\title{
CAMA
}

Centre for Applied Macroeconomic Analysis

\section{Reconciling Output Gaps: Unobserved Components Model and Hodrick-Prescott Filter}

\section{CAMA Working Paper 44/2016 July 2016}

Joshua C.C. Chan

Research School of Economics, ANU and

Centre for Applied Macroeconomic Analysis, ANU

\author{
Angelia L. Grant \\ Research School of Economics, ANU
}

\section{Abstract}

This paper reconciles two widely used trend-cycle decompositions of GDP that give markedly different estimates: the correlated unobserved components model yields output gaps that are small in amplitude, whereas the Hodrick-Prescott (HP) filter generates large and persistent cycles. By embedding the HP filter in an unobserved components model, we show that this difference arises due to differences in the way the stochastic trend is modeled. Moreover, the HP filter implies that the cyclical components are serially independent-an assumption that is decidedly rejected by the data. By relaxing this restrictive assumption, the new model provides comparable model fit relative to the standard correlated unobserved components model. 


\section{Keywords}

trend-cycle decomposition, HP filter, structural break

\section{JEL Classification}

$\mathrm{C} 11, \mathrm{C} 52, \mathrm{E} 32$

\section{Address for correspondence:}

(E) cama.admin@anu.edu.au

\section{ISSN 2206-0332}

The Centre for Applied Macroeconomic Analysis in the Crawford School of Public Policy has been established to build strong links between professional macroeconomists. It provides a forum for quality macroeconomic research and discussion of policy issues between academia, government and the private sector.

The Crawford School of Public Policy is the Australian National University's public policy school, serving and influencing Australia, Asia and the Pacific through advanced policy research, graduate and executive education, and policy impact. 


\title{
Reconciling Output Gaps: Unobserved Components Model and Hodrick-Prescott Filter*
}

\author{
Angelia L. Grant \\ Research School of Economics, \\ Australian National University
}

\author{
Joshua C.C. Chan \\ Research School of Economics, \\ Centre for Applied Macroeconomic Analysis, \\ Australian National University
}

June 2016

\begin{abstract}
This paper reconciles two widely used trend-cycle decompositions of GDP that give markedly different estimates: the correlated unobserved components model yields output gaps that are small in amplitude, whereas the Hodrick-Prescott (HP) filter generates large and persistent cycles. By embedding the HP filter in an unobserved components model, we show that this difference arises due to differences in the way the stochastic trend is modeled. Moreover, the HP filter implies that the cyclical components are serially independent - an assumption that is decidedly rejected by the data. By relaxing this restrictive assumption, the new model provides comparable model fit relative to the standard correlated unobserved components model.
\end{abstract}

Keywords: trend-cycle decomposition, HP filter, structural break

JEL classification: C11, C52, E32

*We thank seminar participants at Australian National University, University of Queensland and University of New South Wales. In particular, this paper has benefited from the discussion with James Morley and Benjamin Wong. Joshua Chan would also like to acknowledge financial support by the Australian Research Council via a Discovery Early Career Researcher Award (DE150100795). 


\section{Introduction}

The estimation of the output gap - the deviation of the output of an economy from its potential or trend output - is an important problem for both academics and policymakers. A particularly important task is to reconcile the differences between estimation methods, given that different approaches provide vastly different results. Morley, Nelson, and Zivot (2003) make an important contribution to such reconciliations. They show that the difference between two widely used trend-cycle decompositions - the Beveridge and Nelson (1981) decomposition and the unobserved components (UC) model of Watson (1986) - is entirely due to one restriction imposed in the UC model: the correlation between the innovations to the trend and cycle is assumed to be zero. When this restriction is relaxed, they find that the two trend-cycle decompositions are identical. In particular, both methods yield output gaps that are noisy and small in amplitude.

However, output gap estimates produced by the popular Hodrick and Prescott (1980, 1997) decomposition are often large and highly persistent. For example, the HodrickPrescott (HP) filter gives an estimate of the US output gap that is as large as $-3 \%$ at the trough of the Great Recession. In contrast, the corresponding estimate from the correlated UC model of Morley et al. (2003) is close to zero. To reconcile the differences, Kamber, Morley, and Wong (2016) investigate how one can generate large cyclical components using the Beveridge-Nelson decomposition, which typically gives small and noisy cycles. They find that by setting the noise-to-signal ratio to be large, instead of estimating it from the data, the cycles obtained are large and the timing of troughs matches the chronology dated by the National Bureau of Economic Research (NBER).

This paper takes an alternative modeling approach to reconciling the small versus large output gaps generated by different methods. We start by embedding the HP filter in a new UC model similar to that in Morley et al. (2003). ${ }^{1}$ By formulating an econometric model for the HP filter and comparing it to other popular UC models, we aim to shed light on the source of differences in the estimated output gaps. We pay particular attention to the assumptions needed to generate large cycles. In addition, since we have an econometric model for the HP filter, we can also use the machinery of model comparison to evaluate which approach fits the data better.

\footnotetext{
${ }^{1}$ The idea of embedding the HP filter in an unobserved components model can be traced back to Harvey and Jaeger (1993). However, they consider an unobserved components model where all the innovations are independent. In contrast, the main purpose of this paper is to reconcile the output gaps obtained from the HP filter and the correlated UC model of Morley et al. (2003).
} 
We begin with the correlated UC model of Morley et al. (2003) in which the trend follows a random walk process with drift. Then, in order to reconcile the small versus large output gaps produced by different estimation methods, we consider a second-order Markov process instead of the random walk specification (which is a first-order Markov process). This modification is useful for our purpose because a higher-order Markov process would imply a smoother trend that does not trace out the actual output as closely. Consequently, the resulting cyclical components would have to be larger. Moreover, we show that using this model, one can recover the HP trend as a Bayesian estimator with a certain dogmatic prior. In particular, we demonstrate that the HP filter implicitly assumes that the cyclical components are serially independent, and this implicit assumption is strongly rejected by the data.

The estimation results, using US real GDP data, confirm that the output gap estimated under the new specification is indeed substantially larger in magnitude than that obtained from the correlated UC model of Morley et al. (2003). Given that the HP filter can be embedded into this new model specification, this suggests that the difference between the output gaps obtained from the correlated UC model and the HP filter arises due to differences in the way trend output is modeled. Furthermore, by allowing the cyclical components to be serially correlated, the output gap estimate from the new model specification is larger and more persistent than the HP filter estimate.

Unlike the correlated UC model, which assumes a constant trend growth rate, this new specification allows for time-varying trend growth. In the application, we show that there is substantial time variation in the trend GDP growth rate. Specifically, the annualized trend growth rate fluctuates between $3.5 \%$ and $4 \%$ from 1950 to 1970; remains stable at about $3 \%$ from the mid-1970s to 2000 ; and starts a gradual decline to about $1.7 \%$ in the middle of the Great Recession. These estimates are comparable to those reported in Perron and Wada (2009) and Grant and Chan (2016) - all obtained using UC models with breaks in the trend growth rate.

In contrast, the annualized trend growth rate implied by the HP filter displays conspicuous cyclical patterns: e.g., it drops from about $4 \%$ in 1985 to about $2.4 \%$ in 1990 , only to rebound again to $4 \%$ in 1997 . Due to the implicit assumption of the HP filter that the cyclical components are serially independent, part of the business cycle variation is absorbed by the time-varying trend growth, resulting in its counter-intuitive large time-variation. In addition, we show in a model comparison exercise that the data overwhelmingly favor the new specification against the HP filter. 
In this paper we focus on the trend-cycle decomposition of real GDP, but our new modeling approach has broader implications. The HP filter continues to be popular, despite concerns that using it to remove low-frequency movements in the data may lead to poor model fit and forecasts (see, e.g., Morley and Piger, 2012; Baştürk, Çakmakli, Ceyhan, and Van Dijk, 2014; Canova, 2014, among many others). It is therefore useful to provide an alternative model-based approach that generalizes the HP filter to accommodate salient features of the data. In addition, using a model-based approach also allows us to formally assess the model fit and compare it with other models.

The rest of this article is organized as follows. Section 2 first discusses the correlated UC model of Morley et al. (2003). It then introduces the new specification with a second-order Markov process for the trend, followed by a brief discussion of the Bayesian estimation methods. We then show that the HP filter trend can be recovered as a posterior mean under the new specification with a dogmatic prior. In Section 3 we report the output gap estimate of the new specification and compare it to the model of Morley et al. (2003) and the HP filter. The trend growth rate and parameter estimates are also reported. Moreover, we compare the performance of the various models in fitting US real GDP. Finally, Section 4 concludes and briefly discusses some future research directions.

\section{UC Model with a Smoother Trend}

The trend-cycle decomposition of aggregate output is motivated by the idea that it can be usefully viewed as the sum of two separate components, namely, a nonstationary component that represents the trend and a transitory deviation from the trend. Based on the correlated unobserved components model of Morley et al. (2003), consider the decomposition of the log real GDP $y_{t}$ :

$$
y_{t}=\tau_{t}+c_{t}
$$

where $\tau_{t}$ is the trend and $c_{t}$ is the stationary, cyclical component. The nonstationary trend $\tau_{t}$ is modeled as a random walk with drift, whereas the cyclical component $c_{t}$ is modeled as a zero mean stationary $\operatorname{AR}(p)$ process:

$$
\begin{aligned}
c_{t} & =\phi_{1} c_{t-1}+\cdots+\phi_{p} c_{t-p}+u_{t}^{c}, \\
\tau_{t} & =\mu+\tau_{t-1}+\widetilde{u}_{t}^{\tau},
\end{aligned}
$$


where the innovations $u_{t}^{c}$ and $\widetilde{u}_{t}^{\tau}$ are jointly normal

$$
\left(\begin{array}{c}
u_{t}^{c} \\
\widetilde{u}_{t}^{\tau}
\end{array}\right) \sim \mathcal{N}\left(\mathbf{0},\left(\begin{array}{cc}
\sigma_{c}^{2} & \rho \sigma_{c} \widetilde{\sigma}_{\tau} \\
\rho \sigma_{c} \widetilde{\sigma}_{\tau} & \widetilde{\sigma}_{\tau}^{2}
\end{array}\right)\right) .
$$

Note that the innovation $\widetilde{u}_{t}^{\tau}$ impacts the level of the trend output $\tau_{t}$. We refer to this model as UCUR. The drift $\mu$ can be interpreted as the average growth rate of trend GDP. Morley et al. (2003) show that the trend-cycle decomposition from this correlated unobserved components model is equivalent to the one obtained from the Beveridge and Nelson (1981) decomposition using an unrestricted ARIMA model-both attribute most of the variance in output to the variation in trend and the cyclical component is small in amplitude.

A limitation of the model is that the trend growth rate $\mu$ is a constant, which is not supported by the data. For example, using US real GDP from 1947Q1 to 1998Q2, Perron and Wada (2009) find a break in trend growth at 1973Q1. Using more recent data, Luo and Startz (2014) and Grant and Chan (2016) find a break at 2006Q1 and 2007Q1, respectively. One way to relax this restrictive assumption is to allow for a time-varying growth rate, as considered in Harvey (1985) and Clark (1987), replacing (3) by

$$
\begin{aligned}
\tau_{t} & =\mu_{t}+\tau_{t-1}+v_{t}^{\tau}, \\
\mu_{t} & =\mu_{t-1}+v_{t}^{\mu} .
\end{aligned}
$$

Under this model, the innovation $v_{t}^{\tau}$ affects the level of the trend output $\tau_{t}$-it plays the role of $\widetilde{u}_{t}^{\tau}$ in the UCUR model-whereas $v_{t}^{\mu}$ changes the trend growth $\mu_{t}$.

While this variant allows for a time-varying growth rate, Perron and Wada (2009, p. 751) conclude that "this generalization leaves the trend-cycle decompositions virtually unchanged". Hence, this model is unable to generate output gaps as large as those from the HP filter. In the next section we consider a variation of the correlated unobserved components model that allows for both a stochastic trend growth and a larger cyclical component.

\subsection{An Alternative Specification for the Trend Component}

Here we consider an alternative unobserved components model that has a new trend specification, with the goal of providing a formal modeling framework for the Hodrick- 
Prescott (HP) filter. To keep our specification as similar as possible to Morley et al. (2003), we only modify the state equation for $\tau_{t}$. In particular, instead of the random walk process with drift in (3), we consider the following second-order Markov process: ${ }^{2}$

$$
\Delta \tau_{t}=\Delta \tau_{t-1}+u_{t}^{\tau}
$$

where $\Delta$ is the first difference operator such that $\Delta x_{t}=x_{t}-x_{t-1}$. As before we assume the innovations $u_{t}^{c}$ and $u_{t}^{\tau}$ are jointly normal

$$
\left(\begin{array}{c}
u_{t}^{c} \\
u_{t}^{\tau}
\end{array}\right) \sim \mathcal{N}\left(\mathbf{0},\left(\begin{array}{cc}
\sigma_{c}^{2} & \rho \sigma_{c} \sigma_{\tau} \\
\rho \sigma_{c} \sigma_{\tau} & \sigma_{\tau}^{2}
\end{array}\right)\right) .
$$

Since $\Delta \tau_{t}$ can be interpreted as the GDP trend growth at time $t$, the specification in $(7)$ implies that the growth rate follows a random walk. As such, by construction the new unobserved components model incorporates a stochastic trend growth rate, in contrast to the constant trend growth rate implied by (3). The former specification is more flexible and can accommodate breaks in GDP growth found in some recent studies such as Perron and Wada (2009) and Luo and Startz (2014).

We emphasize that the new model differs from the correlated unobserved components model in Morley et al. (2003) in only the state equation for $\tau_{t}$. That is, the proposed model is defined by (1), (2), (7) and (8). In addition, we follow Morley et al. (2003) and set $p=2$. Furthermore, the initial values $\tau_{-1}$ and $\tau_{0}$ are treated as parameters to be estimated, and for simplicity we assume that $c_{-1}=c_{0}=0$.

It is instructive to compare the second-order Markov transition for the trend in (7) to the specification in (5)-(6), which also allows for a time-varying growth rate. After some algebra, one can show that the latter specification implies

$$
\Delta \tau_{t}-\Delta \tau_{t-1}=v_{t}^{\mu}+v_{t}^{\tau}-v_{t-1}^{\tau}
$$

i.e., the change in trend growth follows a moving average process. The proposed specification in (7) can therefore be viewed as a limiting case when the variance of $v_{t}^{\tau}$ goes to zero. In other words, the new specification classifies all permanent shocks as shocks to

\footnotetext{
${ }^{2}$ Note that (7) can be written as $\tau_{t}=2 \tau_{t-1}-\tau_{t-2}+u_{t}^{\tau}$, hence it is a second-order Markov process. Although formulated differently, this trend specification can be shown to be equivalent to the one in Harvey, Trimbur, and Van Dijk (2007). The cyclical component in their model, however, is different from ours and the associated cycle estimates from both models are drastically different. For example, their estimated trend growth rate of US GDP does not exhibit any discernible long-term trend over the period from 1950s to 2006, whereas ours shows a gradual downward trend.
} 
the trend growth.

In addition, we show in Section 2.3 that under this new model, one can recover the popular Hodrick and Prescott $(1980,1997)$ decomposition as a Bayesian estimator with a dogmatic prior.

\subsection{Bayesian Estimation}

In this section we outline the Bayesian estimation method used to fit the unobserved components model with the second-order Markov trend transition given in (7). This builds upon the posterior simulator developed in Grant and Chan (2016) for fitting the correlated unobserved components model in Morley et al. (2003). A key novel feature of our approach is that it draws on recent advances in band matrix algorithms developed in Chan and Jeliazkov (2009), McCausland, Miller, and Pelletier (2011) and Chan (2013), which are shown to be more efficient than the conventional Kalman filter-based algorithms. Moreover, due to the modular nature of Markov chain Monte Carlo (MCMC) algorithms, it is relatively straightforward to extend the estimation method to models with unknown breaks or non-Gaussian innovations.

Below we briefly discuss the sampling step for drawing the latent trends $\boldsymbol{\tau}=\left(\tau_{1}, \ldots, \tau_{T}\right)^{\prime}$ and leave the technical details of other steps to the Appendix. This sampling step for drawing $\boldsymbol{\tau}$ is then used in the next section to show how one can recover the HP trend as the posterior mean of $\boldsymbol{\tau}$ with a certain dogmatic prior.

Stack $\mathbf{y}=\left(y_{1}, \ldots, y_{T}\right)^{\prime}$, and similarly define $\mathbf{c}, \mathbf{u}^{c}$ and $\mathbf{u}^{\tau}$. Then, we rewrite the system (1), (2) and (7) in the following matrix form:

$$
\begin{aligned}
\mathbf{y} & =\boldsymbol{\tau}+\mathbf{c}, \\
\mathbf{H}_{\phi} \mathbf{c} & =\mathbf{u}^{c}, \\
\mathbf{H}_{2} \boldsymbol{\tau} & =\widetilde{\boldsymbol{\alpha}}+\mathbf{u}^{\tau},
\end{aligned}
$$


where $\widetilde{\boldsymbol{\alpha}}=\left(2 \tau_{0}-\tau_{-1},-\tau_{0}, 0, \ldots, 0\right)^{\prime}$ and

$$
\mathbf{H}_{2}=\left(\begin{array}{cccccc}
1 & 0 & 0 & 0 & \cdots & 0 \\
-2 & 1 & 0 & 0 & \cdots & 0 \\
1 & -2 & 1 & 0 & \cdots & 0 \\
0 & 1 & -2 & 1 & \cdots & 0 \\
\vdots & \ddots & \ddots & \ddots & \ddots & 0 \\
0 & \cdots & 0 & 1 & -2 & 1
\end{array}\right), \quad \mathbf{H}_{\phi}=\left(\begin{array}{cccccc}
1 & 0 & 0 & 0 & \cdots & 0 \\
-\phi_{1} & 1 & 0 & 0 & \cdots & 0 \\
-\phi_{2} & -\phi_{1} & 1 & 0 & \cdots & 0 \\
0 & -\phi_{2} & -\phi_{1} & 1 & \cdots & 0 \\
\vdots & \ddots & \ddots & \ddots & \ddots & 0 \\
0 & \cdots & 0 & -\phi_{2} & -\phi_{1} & 1
\end{array}\right) .
$$

Note that both $\mathbf{H}_{2}$ and $\mathbf{H}_{\phi}$ are band matrices with only a few nonzero elements arranged around the main diagonal. Further, since both are square matrices with unit determinant, they are invertible. Hence, given the parameters $\phi, \sigma_{c}^{2}, \sigma_{\tau}^{2}, \rho, \tau_{0}$ and $\tau_{-1}$, it follows from (4) that we have

$$
\left(\begin{array}{l}
\mathbf{c} \\
\boldsymbol{\tau}
\end{array}\right) \sim \mathcal{N}\left(\left(\begin{array}{c}
\mathbf{0} \\
\boldsymbol{\alpha}
\end{array}\right),\left(\begin{array}{cc}
\sigma_{c}^{2}\left(\mathbf{H}_{\boldsymbol{\phi}}^{\prime} \mathbf{H}_{\boldsymbol{\phi}}\right)^{-1} & \rho \sigma_{c} \sigma_{\tau}\left(\mathbf{H}_{2}^{\prime} \mathbf{H}_{\boldsymbol{\phi}}\right)^{-1} \\
\rho \sigma_{c} \sigma_{\tau}\left(\mathbf{H}_{\boldsymbol{\phi}}^{\prime} \mathbf{H}_{2}\right)^{-1} & \sigma_{\tau}^{2}\left(\mathbf{H}_{2}^{\prime} \mathbf{H}_{2}\right)^{-1}
\end{array}\right)\right)
$$

where $\boldsymbol{\alpha}=\mathbf{H}_{2}^{-1} \widetilde{\boldsymbol{\alpha}}$. Using the properties of the Gaussian distributions (see, e.g., Kroese and Chan, 2014, Chapter 3.6), the marginal distribution of $\boldsymbol{\tau}$ (unconditional on $\mathbf{c}$ ) is

$$
\left(\boldsymbol{\tau} \mid \sigma_{\tau}^{2}, \tau_{0}, \tau_{-1}\right) \sim \mathcal{N}\left(\boldsymbol{\alpha}, \sigma_{\tau}^{2}\left(\mathbf{H}_{2}^{\prime} \mathbf{H}_{2}\right)^{-1}\right)
$$

and the conditional distribution of $\mathbf{y}$ given $\boldsymbol{\tau}$ and other parameters is given by

$$
\left(\mathbf{y} \mid \boldsymbol{\tau}, \boldsymbol{\phi}, \sigma_{c}^{2}, \sigma_{\tau}^{2}, \rho, \tau_{0}, \tau_{-1}\right) \sim \mathcal{N}\left(\mathbf{H}_{\boldsymbol{\phi}}^{-1} \mathbf{a}+\mathbf{H}_{\boldsymbol{\phi}}^{-1} \mathbf{B} \boldsymbol{\tau},\left(1-\rho^{2}\right) \sigma_{c}^{2}\left(\mathbf{H}_{\phi}^{\prime} \mathbf{H}_{\boldsymbol{\phi}}\right)^{-1}\right)
$$

where

$$
\mathbf{a}=-\frac{\rho \sigma_{c}}{\sigma_{\tau}} \mathbf{H}_{2} \boldsymbol{\alpha}, \quad \mathbf{B}=\mathbf{H}_{\phi}+\frac{\rho \sigma_{c}}{\sigma_{\tau}} \mathbf{H}_{2}
$$

Therefore, the prior density of $\boldsymbol{\tau}$ and the conditional likelihood are given by

$$
\begin{gathered}
p\left(\boldsymbol{\tau} \mid \sigma_{\tau}^{2}, \tau_{0}, \tau_{-1}\right)=\left(2 \pi \sigma_{\tau}^{2}\right)^{-\frac{T}{2}} \mathrm{e}^{-\frac{1}{2 \sigma_{\tau}^{2}}(\boldsymbol{\tau}-\boldsymbol{\alpha})^{\prime} \mathbf{H}_{2}^{\prime} \mathbf{H}_{2}(\boldsymbol{\tau}-\boldsymbol{\alpha})} \\
p\left(\mathbf{y} \mid \boldsymbol{\tau}, \boldsymbol{\phi}, \sigma_{c}^{2}, \sigma_{\tau}^{2}, \rho, \tau_{0}, \tau_{-1}\right)=\left(2 \pi \sigma_{y}^{2}\left(1-\rho^{2}\right)\right)^{-\frac{T}{2}} \mathrm{e}^{-\frac{1}{2\left(1-\rho^{2}\right) \sigma_{c}^{2}}\left(\mathbf{H}_{\phi} \mathbf{y}-\mathbf{a}-\mathbf{B} \boldsymbol{\tau}\right)^{\prime}\left(\mathbf{H}_{\phi} \mathbf{y}-\mathbf{a}-\mathbf{B} \boldsymbol{\tau}\right)}
\end{gathered}
$$

Then, by standard linear regression results (see, e.g., Kroese and Chan, 2014, p.237-240), we have

$$
\left(\boldsymbol{\tau} \mid \mathbf{y}, \boldsymbol{\phi}, \sigma_{c}^{2}, \sigma_{\tau}^{2}, \rho, \tau_{0}, \tau_{-1}\right) \sim \mathcal{N}\left(\widehat{\boldsymbol{\tau}}, \mathbf{K}_{\boldsymbol{\tau}}^{-1}\right)
$$


where

$$
\mathbf{K}_{\boldsymbol{\tau}}=\frac{1}{\sigma_{\tau}^{2}} \mathbf{H}_{2}^{\prime} \mathbf{H}_{2}+\frac{1}{\left(1-\rho^{2}\right) \sigma_{c}^{2}} \mathbf{B}^{\prime} \mathbf{B}, \quad \widehat{\boldsymbol{\tau}}=\mathbf{K}_{\boldsymbol{\tau}}^{-1}\left(\frac{1}{\sigma_{\tau}^{2}} \mathbf{H}_{2}^{\prime} \mathbf{H}_{2} \boldsymbol{\alpha}+\frac{1}{\left(1-\rho^{2}\right) \sigma_{c}^{2}} \mathbf{B}^{\prime}\left(\mathbf{H}_{\phi} \mathbf{y}-\mathbf{a}\right)\right) .
$$

Since $\mathbf{H}_{2}, \mathbf{H}_{\boldsymbol{\phi}}$ and $\mathbf{B}$ are all band matrices, so is the precision matrix $\mathbf{K}_{\boldsymbol{\tau}}$. As such, the precision sampler of Chan and Jeliazkov (2009) can be used to sample $\boldsymbol{\tau}$ efficiently.

We assume proper but relatively noninformative priors for the model parameters $\phi=$ $\left(\phi_{1}, \phi_{2}\right)^{\prime}, \sigma_{c}^{2}, \sigma_{\tau}^{2}, \rho, \tau_{0}$ and $\tau_{-1}$. In particular, we consider uniform priors for $\rho, \sigma_{c}^{2}$ and $\sigma_{\tau}^{2}$. The details of the priors and the steps for the rest of the posterior sampler are contained in the Appendix.

In the next section, we show that the conditional mean of $\boldsymbol{\tau}$ given in (11) reduces to the Hodrick-Prescott decomposition if one assumes a certain dogmatic prior for the model parameters.

\subsection{HP Decomposition as a Dogmatic Bayesian Estimator}

Recall that the Hodrick-Prescott decomposition is formulated as a minimization problem in which the HP trend is the minimizer of the following smoothed square loss:

$$
\widehat{\boldsymbol{\tau}}_{\mathrm{HP}} \equiv \underset{\boldsymbol{\tau}}{\operatorname{argmin}}\left[\sum_{t=1}^{T}\left(y_{t}-\tau_{t}\right)^{2}+\lambda \sum_{t=1}^{T}\left(\Delta \tau_{t}-\Delta \tau_{t-1}\right)^{2}\right],
$$

where $\lambda$ is a fixed constant that penalizes variability in the trend component. The larger the value of $\lambda$, the smoother is the associated HP trend $\widehat{\tau}_{\mathrm{HP}}$. Hodrick and Prescott (1980, 1997) highlight that $\lambda$ may be viewed as the noise-to-signal ratio under certain restrictive conditions - namely, that "the cyclical components and the second differences of the [trend] components were identically and independently distributed." They then suggest setting $\lambda=1600$ for US quarterly data.

Below we show that the HP trend $\widehat{\boldsymbol{\tau}}_{\text {HP }}$ can be viewed as a posterior mean of $\boldsymbol{\tau}$ under the 
model defined in (1), (2) and (7). ${ }^{3}$ To that end, first note that

$$
\sum_{t=1}^{T}\left(\Delta \tau_{t}-\Delta \tau_{t-1}\right)^{2}=\left(\mathbf{H}_{2} \boldsymbol{\tau}-\widetilde{\boldsymbol{\alpha}}\right)^{\prime}\left(\mathbf{H}_{2} \boldsymbol{\tau}-\widetilde{\boldsymbol{\alpha}}\right)=(\boldsymbol{\tau}-\boldsymbol{\alpha})^{\prime} \mathbf{H}_{2}^{\prime} \mathbf{H}_{2}(\boldsymbol{\tau}-\boldsymbol{\alpha})
$$

where $\boldsymbol{\alpha}=\mathbf{H}_{2}^{-1} \widetilde{\boldsymbol{\alpha}}$ and $\widetilde{\boldsymbol{\alpha}}=\left(2 \tau_{0}-\tau_{-1},-\tau_{0}, 0, \ldots, 0\right)^{\prime}$. Hence, the minimization problem can be rewritten as

$$
\widehat{\boldsymbol{\tau}}_{\mathrm{HP}} \equiv \underset{\boldsymbol{\tau}}{\operatorname{argmin}}\left[(\mathbf{y}-\boldsymbol{\tau})^{\prime}(\mathbf{y}-\boldsymbol{\tau})+\lambda(\boldsymbol{\tau}-\boldsymbol{\alpha})^{\prime} \mathbf{H}_{2}^{\prime} \mathbf{H}_{2}(\boldsymbol{\tau}-\boldsymbol{\alpha})\right]
$$

By differentiating the objective function with respect to $\boldsymbol{\tau}$, we obtain the following firstorder condition:

$$
\left.\frac{\partial}{\partial \boldsymbol{\tau}}\left[\boldsymbol{\tau}^{\prime} \boldsymbol{\tau}-2 \boldsymbol{\tau}^{\prime} \mathbf{y}+\lambda \boldsymbol{\tau}^{\prime} \mathbf{H}_{2}^{\prime} \mathbf{H}_{2} \boldsymbol{\tau}-2 \lambda \boldsymbol{\tau}^{\prime} \mathbf{H}_{2}^{\prime} \mathbf{H}_{2} \boldsymbol{\alpha}\right)\right]=2\left(\mathbf{I}_{T}+\lambda \mathbf{H}_{2}^{\prime} \mathbf{H}_{2}\right) \boldsymbol{\tau}-2\left(\mathbf{y}+\lambda \mathbf{H}_{2}^{\prime} \mathbf{H}_{2} \boldsymbol{\alpha}\right)
$$

Setting the first-order condition to zero and solving for $\boldsymbol{\tau}$, we obtain

$$
\widehat{\boldsymbol{\tau}}_{\mathrm{HP}}=\left(\mathbf{I}_{T}+\lambda \mathbf{H}_{2}^{\prime} \mathbf{H}_{2}\right)^{-1}\left(\mathbf{y}+\lambda \mathbf{H}_{2}^{\prime} \mathbf{H}_{2} \boldsymbol{\alpha}\right)
$$

Since the Hessian is $2\left(\mathbf{I}_{T}+\lambda \mathbf{H}_{2}^{\prime} \mathbf{H}_{2}\right)$, which is always positive definite provided that $\lambda>0$, $\widehat{\boldsymbol{\tau}}_{\mathrm{HP}}$ is the unique minimizer. Also, the expression (12) provides a quick way to compute the HP trend as all $T \times T$ matrices are banded.

Next, we show that $\widehat{\boldsymbol{\tau}}_{\mathrm{HP}}$ is the mean of the conditional distribution of $\boldsymbol{\tau}$ given in (11). To see that, set $\rho=0$ and $\boldsymbol{\phi}=\mathbf{0}$. Then, the posterior mean of $\boldsymbol{\tau}$ becomes

$$
\begin{aligned}
\widehat{\boldsymbol{\tau}} & =\left(\frac{1}{\sigma_{\tau}^{2}} \mathbf{H}_{2}^{\prime} \mathbf{H}_{2}+\frac{1}{\sigma_{c}^{2}} \mathbf{I}_{T}\right)^{-1}\left(\frac{1}{\sigma_{\tau}^{2}} \mathbf{H}_{2}^{\prime} \mathbf{H}_{2} \boldsymbol{\alpha}+\frac{1}{\sigma_{c}^{2}} \mathbf{y}\right) \\
& =\left(\frac{\sigma_{c}^{2}}{\sigma_{\tau}^{2}} \mathbf{H}_{2}^{\prime} \mathbf{H}_{2}+\mathbf{I}_{T}\right)^{-1}\left(\frac{\sigma_{c}^{2}}{\sigma_{\tau}^{2}} \mathbf{H}_{2}^{\prime} \mathbf{H}_{2} \boldsymbol{\alpha}+\mathbf{y}\right)=\widehat{\boldsymbol{\tau}}_{\mathrm{HP}}
\end{aligned}
$$

with $\lambda=\sigma_{c}^{2} / \sigma_{\tau}^{2}$. Hence, we have proved the following proposition.

\footnotetext{
${ }^{3}$ It is unclear whether Hodrick and Prescott $(1980,1997)$ had a statistical model in mind when they proposed the Hodrick-Prescott decomposition. On the one hand, they realized that their minimization problem can be solved by the Kalman filter-hence, it has a state space form. On the other hand, they explicitly stated that "[o] ur statistical approach does not utilize standard time series analysis. Our prior knowledge concerning the process generating the data is not of the variety that permits us to specify a probability model as required for application of that analysis."

Polasek (2011) seems to be the first to realize that the HP trend can be interpreted as a posterior mean under a certain prior for $\boldsymbol{\tau}$. We take this one step further to show that the underlying model is in fact a state space model with a second-order Markov transition for the trend.
} 
Proposition 1. Consider the unobserved components model with a second-order Markov transition defined by (1), (2), (4) and (7). The Hodrick-Prescott trend $\widehat{\boldsymbol{\tau}}_{\mathrm{HP}}$ is the posterior mean of $\boldsymbol{\tau}$ under the dogmatic priors of fixing $\rho=0, \boldsymbol{\phi}=\mathbf{0}$ and $\lambda=\sigma_{c}^{2} / \sigma_{\tau}^{2}$.

It is intriguing that the underlying econometric model of the HP trend requires $\boldsymbol{\phi}=\mathbf{0}$. That is, the cyclical components are assumed to be serially independent. As we will see in the next section, this assumption is strongly rejected by the data.

\section{Empirical Results}

In this section we report the cycle estimates and other parameters of interest under the unobserved components model with a second-order Markov trend transition (UCUR$2 \mathrm{M})$. We compare these estimates with those obtained under standard benchmarks to assess the impact of the new specification on the trend evolution. In particular, we present results from the correlated unobserved components (UCUR) model of Morley et al. (2003). Moreover, given the findings in Perron and Wada (2009), Luo and Startz (2014) and Grant and Chan (2016), we also consider a version in which the trend growth rate has a break at $t_{0}$. More precisely, we replace (3) with

$$
\tau_{t}=\mu\left(t<t_{0}\right)+\gamma 1\left(t \geqslant t_{0}\right)+\tau_{t-1}+\widetilde{u}_{t}^{\tau},
$$

where $1(A)$ is the indicator function that takes the value 1 if the condition $A$ is true and 0 otherwise. In other words, the stochastic trend $\tau_{t}$ has a growth rate of $\mu$ before the break $t_{0}$ and a growth rate of $\gamma$ after the break. We set the break date at 2007Q1 and refer to this model as UCUR-07.

We use US quarterly real GDP from 1947Q1 to 2014Q4 for our analysis. The data are sourced from the Federal Reserve Bank of St. Louis economic database, and the series is then transformed by taking the logs and multiplying by 100. Each set of results below is based on 100000 posterior draws after a burn-in period of 10000 .

\subsection{Output Gap Estimates}

Figure 1 plots the estimates of the output gap $c_{t}$ under the UCUR and UCUR-07 models. It is evident that the estimates from both models are remarkably similar until the 
mid-2000s, when the UCUR-07 model allows for a break in trend growth in 2007Q1. This finding contrasts with the results in Perron and Wada (2009), who find that when a break is allowed for, the estimates from correlated UC models with and without a break differ substantially. One source of difference is that their output gap estimates are computed given the maximum likelihood estimates, thus ignoring parameter uncertainty. In contrast, ours take into account parameter uncertainty by averaging the output gap estimates over posterior draws of the model parameters.

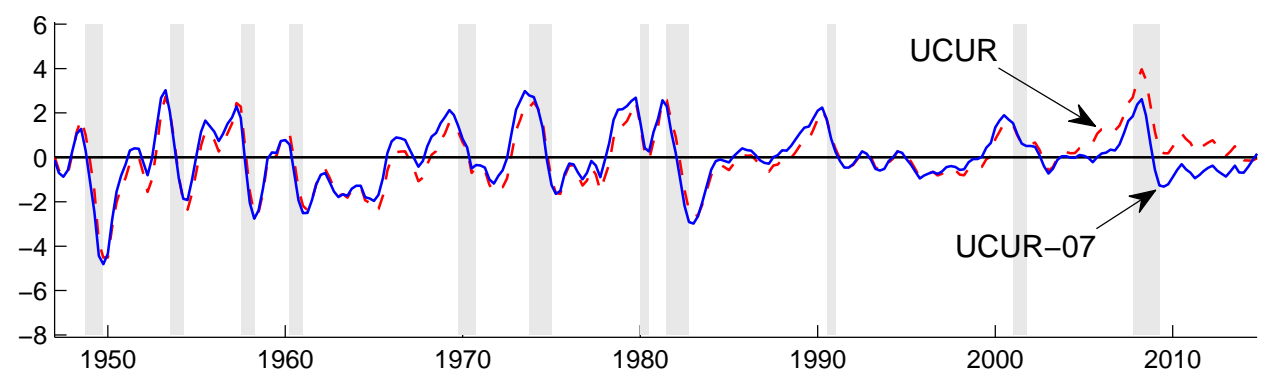

Figure 1: Estimates of the output gap $c_{t}$ under the UCUR and UCUR-07 models. The shaded regions are the NBER recession dates.

The output gap estimates in Figure 1 generally coincide with the NBER recession dates, but they are relatively small. For example, the trough of the 1981-1982 recession is only about $-3 \%$; during the Great Recession the output gap estimates are about $-1.3 \%$ for the UCUR-07 model and close to 0 for the UCUR.

The corresponding estimates under the UCUR-2M model are reported in Figure 2. It is clear that the plot of the output gap has a similar shape as that in Figure 1; it traces the NBER recession dates closely as well. The main difference is that the new output gap estimates are noticeably larger. For instance, the trough of the 1981-1982 recession is now at about $-7 \%$ and the trough in the Great recession is about $-4 \%$.

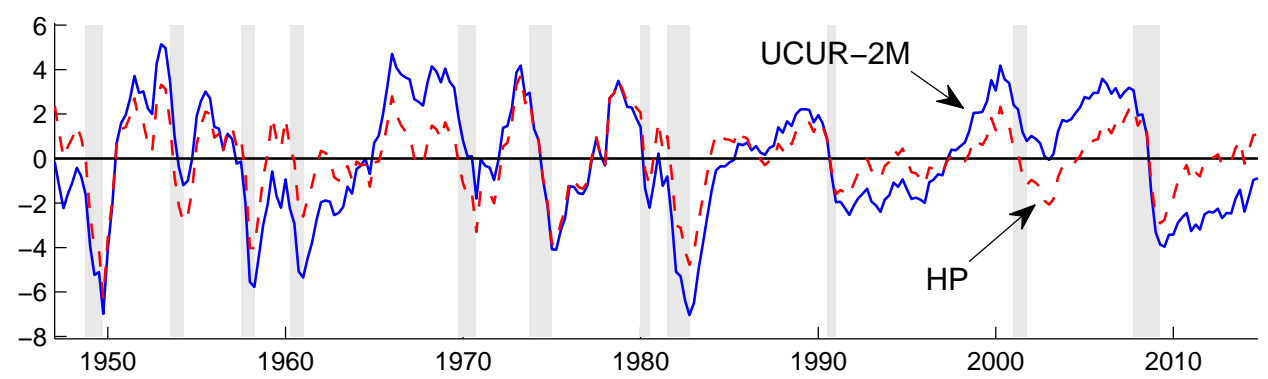

Figure 2: Estimates of the output gap $c_{t}$ under the UCUR-2M model and HP filter. The shaded regions are the NBER recession dates. 
For comparison, we also plot the output estimates obtained from the HP filter in Figure 2. The two plots generally coincide, but there a few noticeable differences. In particular, the output gap estimates under the UCUR-2M model are typically larger and more persistent. For instance, the HP filter estimates drop to 0 in 2011-2012, whereas the UCUR-2M model shows a persistent output gap - it remains around $-0.9 \%$ at $2014 \mathrm{Q} 4$.

This difference is due to the assumption that the cyclical components are independent under the HP filter, i.e., $\boldsymbol{\phi}=\mathbf{0}$. In contrast, when we allow $\boldsymbol{\phi}$ to be estimated as in the UCUR-2M model, the estimates are far from zero (see the estimation results below). This in turn makes the output gap estimate under the UCUR-2M model more persistent.

\subsection{Trend GDP Growth Estimates}

Next, we present the estimated annualized trend GDP growth rate - the posterior means of $4 \Delta \tau_{t}=4\left(\tau_{t}-\tau_{t-1}\right)$-in Figure 3. It is evident that there is substantial time variation in trend growth over the past six decades. In particular, the annualized trend growth rate fluctuates between $3.5 \%$ and $4 \%$ from the beginning of the sample until 1970. It then begins a steady decline and reaches about 3\% in mid-1970s. These estimates are similar to those in Berger, Everaert, and Vierke (2016), who find substantial time variation in trend output growth using a trivariate unobserved components model of output, inflation and unemployment rate.

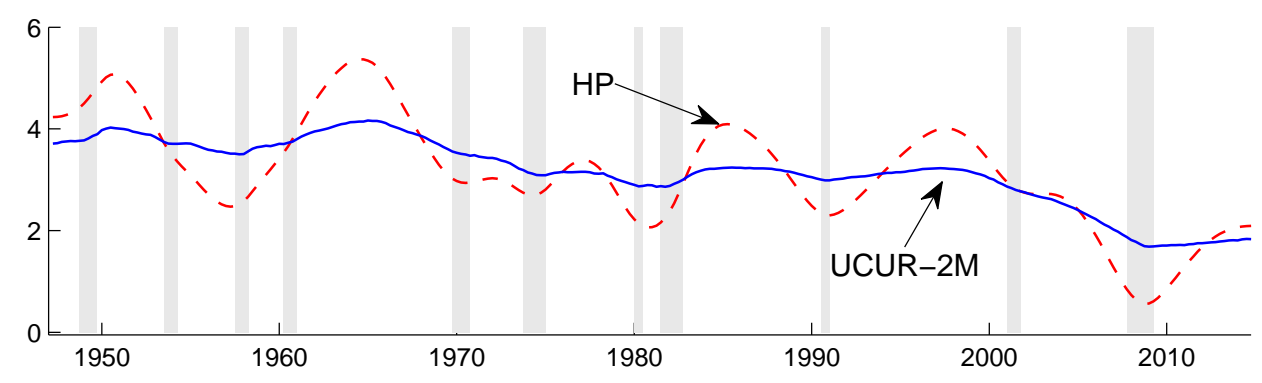

Figure 3: Estimates of the annualized growth in trend $4 \Delta \tau_{t}$ under the UCUR-2M model and HP filter. The shaded regions are the NBER recession dates.

The estimated trend growth rate remains stable at about 3\% from the mid-1970s until 2000, when it starts another gradual decline to about $1.7 \%$ in the middle of the Great Recession. These results are similar to the estimated trend growth rates reported in Grant and Chan (2016) - they find that the trend growth rate drops from $3.4 \%$ before 2007 to 
about $1.5 \%$ afterward. Our estimate is also in line with the forecast of US potential output growth of $1.5 \%$ to $1.55 \%$ from 2007 to 2032 given in Gordon (2014).

For comparison we also plot the annualized trend growth rate implied by the HP filter in Figure 3. The most prominent feature of the plot is its apparent cyclical pattern. For instance, the growth rate drops from about $4 \%$ in 1985 to about $2.4 \%$ in 1990, only to rebound again to $4 \%$ in 1997 . This counter-intuitive large variation in trend growth might reflect the assumption that the cyclical components are independent-resulting in some of the business cycle variation being accommodated by the time-varying trend. In contrast, when we allow the cyclical components to be correlated via an $\operatorname{AR}(2)$ process in the UCUR-2M model, the estimated trend growth rate is much smoother and picks up only some very low-frequency movement.

\subsection{Parameter Estimates and Model Comparison}

Figure 4 presents the contour plot of the bivariate posterior density $p\left(\phi_{1}, \phi_{2} \mid \mathbf{y}\right)$ under the UCUR-2M model. ${ }^{4}$ It is evident that most of the mass of this density is concentrated around $(1.3,-0.4)$ and there is no mass near the origin. This can be viewed as evidence that the assumption $\boldsymbol{\phi}=\mathbf{0}$ implied by the HP filter is strongly rejected by the data. (Below we also perform a formal model comparison.) It is also worth noting that one of the stationarity restrictions, namely, $\phi_{1}+\phi_{2}<1$, appears to be binding, reflecting the fact that the cyclical components are highly persistent.

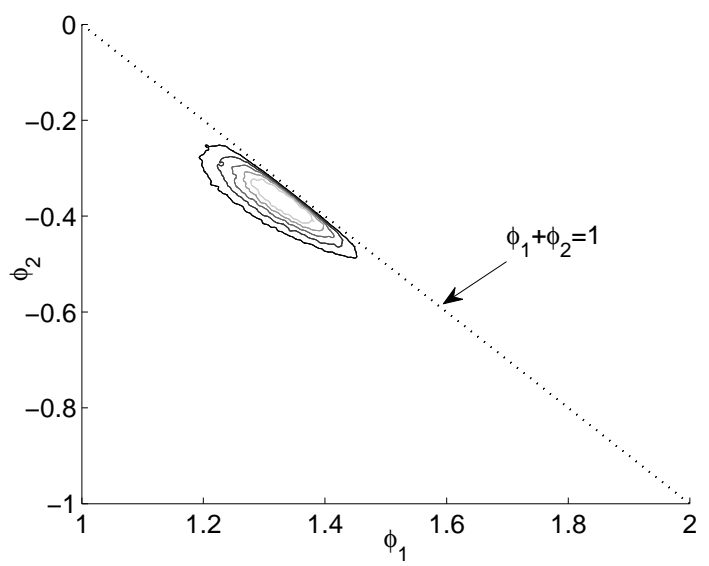

Figure 4: Contour plot of the bivariate posterior density $p\left(\phi_{1}, \phi_{2} \mid \mathbf{y}\right)$.

\footnotetext{
${ }^{4}$ Posterior draws of $\phi=\left(\phi_{1}, \phi_{2}\right)^{\prime}$ are first obtained using the MCMC sampler described in the Appendix. These draws are then used to compute the density using the kernel density estimator of Botev, Grotowski, and Kroese (2010).
} 
Next, we report in Table 1 the parameter estimates under the UCUR-2M model, as well as estimates from the three other models for comparison. Consistent with the contour plot in Figure 4, the posterior means of $\phi_{1}$ and $\phi_{2}$ are estimated to be 1.31 and -0.37 respectively with small standard errors. These estimates are similar to those obtained under the UCUR and UCUR-07 models, highlighting that the cyclical components are highly persistent regardless of how the trend is modeled.

Table 1: Estimated posterior means under UCUR, UCUR-07, UCUR-2M and HP. Numerical standard errors are in parentheses.

\begin{tabular}{ccccc}
\hline \hline & UCUR & UCUR-07 & UCUR-2M & HP \\
\hline$\mu$ & 0.78 & 0.84 & - & - \\
& $(0.08)$ & $(0.08)$ & & - \\
$\gamma$ & - & 0.37 & - & - \\
& & $(0.20)$ & & - \\
$\phi_{1}$ & 0.95 & 1.10 & 1.31 & - \\
& $(0.34)$ & $(0.36)$ & $(0.07)$ & \\
$\phi_{2}$ & -0.36 & -0.44 & -0.37 & - \\
& $(0.18)$ & $(0.18)$ & $(0.06)$ & \\
$\sigma_{c}^{2}$ & 1.12 & 0.90 & 0.76 & 2.30 \\
& $(0.55)$ & $(0.49)$ & $(0.07)$ & $(0.15)$ \\
$\tilde{\sigma}_{\tau}^{2}$ & 1.85 & 1.42 & - & - \\
& $(0.49)$ & $(0.59)$ & & \\
$\sigma_{\tau}^{2}$ & - & - & 0.0028 & - \\
& & & $(0.0023)$ & \\
$\rho$ & -0.87 & -0.76 & 0.00 & - \\
& $(0.07)$ & $(0.25)$ & $(0.55)$ & \\
\hline \hline
\end{tabular}

Under UCUR-2M the estimates of $\sigma_{c}^{2}$ and $\sigma_{\tau}^{2}$ are 0.76 and 0.0028 respectively, giving a ratio of about 271, which is much smaller than the typical value of $\lambda=1600$ used in the HP filter. Despite this smaller noise-to-signal ratio, the estimates of the output gap $c_{t}$ under UCUR-2M reported in Figure 2 are larger in magnitude and more persistent than those obtained from the HP filter. As discussed, this is because under the HP filter the cyclical components are assumed to be independent whereas they are highly persistent under UCUR-2M. It is interesting to note that under UCUR-2M, the estimated $\rho$ is 0 , indicating that the innovations to the cyclical components and the trend growth rate are uncorrelated.

Lastly, we compare the four models in fitting US real GDP in a formal Bayesian model comparison using the marginal likelihood. The marginal likelihood can be interpreted as a density forecast of the data under the model evaluated at the actual observed data. 
Hence, if the observed data are likely under the model, the associated marginal likelihood would be "large". For a more detailed discussion, see, e.g., Koop (2003) and Geweke and Amisano (2011). The marginal likelihoods are computed using the adaptive importance sampling method proposed in Chan and Eisenstat (2015). The results are reported in Table 2.

Table 2: Log marginal likelihoods of competing models. Numerical standard errors are in parentheses.

\begin{tabular}{cccc}
\hline \hline UCUR & UCUR-07 & UCUR-2M & HP \\
\hline-365.0 & -364.0 & -369.8 & -601.5 \\
$(0.03)$ & $(0.04)$ & $(0.02)$ & $(0.31)$ \\
\hline \hline
\end{tabular}

According to the marginal likelihood, the UCUR-07 model with a break in 2007Q1 is the best model, followed by UCUR and UCUR-2M, whereas the HP filter is by far the worst. This supports the conclusion in Morley and Piger (2012, p.214) that the HP filter is "strongly at odds with the data." In addition, the data generally prefer UCUR or UCUR-07 over UCUR-2M, but the evidence is not overwhelming. If the researcher prefers to have larger output gaps, the UCUR-2M model seems to be the best choice - it produces a more persistent output gap at a slight cost of model fit.

\section{Concluding Remarks and Future Research}

We formulate a new correlated unobserved components model with a second-order Markov process and show one can recover the HP trend as a Bayesian estimator with a certain dogmatic prior. Using this model-based approach, we directly compare the HP filter with other popular unobserved components models and shed light on the source of differences in the cycle estimates. We show that by relaxing the implicit assumption that the cyclical components are independent under the HP filter, the new model provides comparable model fit relative to the standard correlated unobserved components model.

Many recent papers, including Carriero, Kapetanios, and Marcellino (2009), Banbura, Giannone, and Reichlin (2010) and Koop (2013), have demonstrated the gains of incorporating the information content in a large number of macroeconomic variables. For future research, it would be interesting to embed the proposed UC model in a large Bayesian vector autoregression to assess if other macroeconomic variables provide additional information about the output gap. 


\section{Appendix: Estimation Details}

This appendix discusses the priors and provides the estimation details of the proposed unobserved components model. The sampler discussed is based on the posterior simulator developed in Grant and Chan (2016) for fitting the correlated unobserved components model in Morley et al. (2003).

We assume proper but relatively noninformative priors for the model parameters $\phi=$ $\left(\phi_{1}, \phi_{2}\right)^{\prime}, \sigma_{c}^{2}, \sigma_{\tau}^{2}, \rho, \tau_{0}$ and $\tau_{-1}$. In particular, we assume independent priors for $\phi, \tau_{0}$ and $\tau_{-1}$ :

$$
\boldsymbol{\phi} \sim \mathcal{N}\left(\phi_{0}, \mathbf{V}_{\phi}\right) 1(\phi \in \mathbf{R}), \quad \tau_{0}, \tau_{-1} \sim \mathcal{N}\left(\tau_{00}, V_{\tau}\right)
$$

where $\mathbf{R}$ is the stationarity region. We assume relatively large prior variances with $\mathbf{V}_{\phi}=\mathbf{I}_{2}$ and $V_{\tau}=100$. For the prior means, we set $\phi_{0}=(1.3,-0.7)^{\prime}$ and $\tau_{00}=750$. In particular, these values imply that the $\mathrm{AR}(2)$ process of the transitory component has two complex roots. Next, $\sigma_{c}^{2}, \sigma_{\tau}^{2}$ and $\rho$ have uniform priors:

$$
\sigma_{c}^{2} \sim \mathcal{U}\left(0, b_{c}\right), \quad \sigma_{\tau}^{2} \sim \mathcal{U}\left(0, b_{\tau}\right), \quad \rho \sim \mathcal{U}(-1,1)
$$

where we set the upper bounds as $b_{c}=3$ and $b_{\tau}=0.01$.

Next, we describe the posterior sampler. Specifically, posterior draws can be obtained by sequentially sampling from the following densities: 1. $p\left(\boldsymbol{\tau} \mid \mathbf{y}, \boldsymbol{\phi}, \sigma_{c}^{2}, \sigma_{\tau}^{2}, \rho, \tau_{0}, \tau_{-1}\right) ; 2$. $p\left(\boldsymbol{\phi} \mid \mathbf{y}, \boldsymbol{\tau}, \sigma_{c}^{2}, \sigma_{\tau}^{2}, \rho, \tau_{0}, \tau_{-1}\right) ; 3 . p\left(\sigma_{c}^{2} \mid \mathbf{y}, \boldsymbol{\tau}, \boldsymbol{\phi}, \sigma_{\tau}^{2}, \rho, \tau_{0}, \tau_{-1}\right) ; 4 . p\left(\sigma_{\tau}^{2} \mid \mathbf{y}, \boldsymbol{\tau}, \boldsymbol{\phi}, \sigma_{c}^{2}, \rho, \tau_{0}, \tau_{-1}\right) ;$ 5. $p\left(\rho \mid \mathbf{y}, \boldsymbol{\tau}, \boldsymbol{\phi}, \sigma_{c}^{2}, \sigma_{\tau}^{2}, \tau_{0}, \tau_{-1}\right)$; and 6. $p\left(\tau_{0}, \tau_{-1} \mid \mathbf{y}, \boldsymbol{\tau}, \boldsymbol{\phi}, \sigma_{c}^{2}, \sigma_{\tau}^{2}, \rho\right)$. The implementation of Step 1 is discussed in Section 2.2. Here we provide the details of the other steps.

To sample $\boldsymbol{\phi}$ in Step 2, recall that $\mathbf{u}^{c}$ and $\boldsymbol{\tau}$ are jointly normal:

$$
\left(\begin{array}{c}
\mathbf{u}^{c} \\
\boldsymbol{\tau}
\end{array}\right) \sim \mathcal{N}\left(\left(\begin{array}{l}
\mathbf{0} \\
\boldsymbol{\alpha}
\end{array}\right),\left(\begin{array}{cc}
\sigma_{c}^{2} \mathbf{I}_{T} & \rho \sigma_{c} \sigma_{\tau}\left(\mathbf{H}_{2}^{\prime}\right)^{-1} \\
\rho \sigma_{c} \sigma_{\tau} \mathbf{H}_{2}^{-1} & \sigma_{\tau}^{2}\left(\mathbf{H}_{2}^{\prime} \mathbf{H}_{2}\right)^{-1}
\end{array}\right)\right)
$$

where $\boldsymbol{\alpha}=\mathbf{H}_{2}^{-1} \widetilde{\boldsymbol{\alpha}}$ with $\widetilde{\boldsymbol{\alpha}}=\left(2 \tau_{0}-\tau_{-1},-\tau_{0}, 0, \ldots, 0\right)^{\prime}$. Hence, the conditional distribution of $\mathbf{u}^{c}$ given $\boldsymbol{\tau}$ and other parameters is

$$
\left(\mathbf{u}^{c} \mid \boldsymbol{\tau}, \sigma_{c}^{2}, \sigma_{\tau}^{2}, \rho, \tau_{0}, \tau_{-1}\right) \sim \mathcal{N}\left(\frac{\rho \sigma_{c}}{\sigma_{\tau}} \mathbf{H}_{2}(\boldsymbol{\tau}-\boldsymbol{\alpha}),\left(1-\rho^{2}\right) \sigma_{c}^{2} \mathbf{I}_{T}\right)
$$

Next, we write (2) as

$$
\mathbf{c}=\mathbf{X}_{\phi} \phi+\mathbf{u}^{c}
$$


where $\mathbf{X}_{\phi}$ is a $T \times 2$ matrix consisting of lagged values of $c_{t}$. Then, by standard regression results, we have

$$
\left(\boldsymbol{\phi} \mid \mathbf{y}, \boldsymbol{\tau}, \sigma_{c}^{2}, \sigma_{\tau}^{2}, \rho, \tau_{0}, \tau_{-1}\right) \sim \mathcal{N}\left(\widehat{\phi}, \mathbf{K}_{\phi}^{-1}\right) 1(\boldsymbol{\phi} \in \mathbf{R})
$$

where

$$
\begin{aligned}
\mathbf{K}_{\phi} & =\mathbf{V}_{\phi}^{-1}+\frac{1}{\left(1-\rho^{2}\right) \sigma_{c}^{2}} \mathbf{X}_{\phi}^{\prime} \mathbf{X}_{\phi}, \\
\widehat{\boldsymbol{\phi}} & =\mathbf{K}_{\phi}^{-1}\left(\mathbf{V}_{\phi}^{-1} \boldsymbol{\phi}_{0}+\frac{1}{\left(1-\rho^{2}\right) \sigma_{c}^{2}} \mathbf{X}_{\boldsymbol{\phi}}^{\prime}\left(\mathbf{c}-\frac{\rho \sigma_{c}}{\sigma_{\tau}} \mathbf{H}_{2}(\boldsymbol{\tau}-\boldsymbol{\alpha})\right)\right) .
\end{aligned}
$$

A draw from this truncated normal distribution can be obtained by the acceptancerejection method, i.e., keep sampling from $\mathcal{N}\left(\widehat{\phi}, \mathbf{K}_{\phi}^{-1}\right)$ until $\phi \in \mathbf{R}$.

To implement Steps 3 to 5 , we first derive the joint density of $\mathbf{u}^{c}$ and $\mathbf{u}^{\tau}$. To that end, note that given $\sigma_{c}^{2}, \sigma_{\tau}^{2}$ and $\rho$, we can factorize $\left(u_{t}^{c}, u_{t}^{\tau}\right)$ as:

$$
u_{t}^{\tau} \sim \mathcal{N}\left(0, \sigma_{\tau}^{2}\right), \quad\left(u_{t}^{c} \mid u_{t}^{\tau}\right) \sim \mathcal{N}\left(\frac{\rho \sigma_{c}}{\sigma_{\tau}} u_{t}^{\tau},\left(1-\rho^{2}\right) \sigma_{c}^{2}\right)
$$

Hence, the joint density of $\mathbf{u}^{c}$ and $\mathbf{u}^{\tau}$ is given by

$$
\begin{aligned}
p\left(\mathbf{u}^{c}, \mathbf{u}^{\tau} \mid \sigma_{c}^{2}, \sigma_{\tau}^{2}, \rho\right) & \propto\left(\sigma_{\tau}^{2}\right)^{-\frac{T}{2}} \mathrm{e}^{-\frac{1}{2 \sigma_{\tau}^{2}} \sum_{t=1}^{T}\left(u_{t}^{\tau}\right)^{2}}\left(\left(1-\rho^{2}\right) \sigma_{c}^{2}\right)^{-\frac{T}{2}} \mathrm{e}^{-\frac{1}{2\left(1-\rho^{2}\right) \sigma_{c}^{2}} \sum_{t=1}^{T}\left(u_{t}^{c}-\frac{\rho \sigma_{c}}{\sigma \tau} u_{t}^{\tau}\right)^{2}}, \\
& =\left(\left(1-\rho^{2}\right) \sigma_{c}^{2} \sigma_{\tau}^{2}\right)^{-\frac{T}{2}} \mathrm{e}^{-\frac{1}{2 \sigma_{\tau}^{2}} k_{3}-\frac{1}{2\left(1-\rho^{2}\right) \sigma_{c}^{2}}\left(k_{1}-\frac{2 \rho \sigma_{c}}{\sigma_{\tau}} k_{2}+\frac{\rho^{2} \sigma_{c}^{2}}{\sigma_{\tau}^{2}} k_{3}\right),}
\end{aligned}
$$

where $k_{1}=\sum_{t=1}^{T}\left(u_{t}^{c}\right)^{2}, k_{2}=\sum_{t=1}^{T} u_{t}^{c} u_{t}^{\tau}$ and $k_{3}=\sum_{t=1}^{T}\left(u_{t}^{\tau}\right)^{2}$. It follows from (14) that

$$
p\left(\sigma_{c}^{2} \mid \mathbf{y}, \boldsymbol{\tau}, \boldsymbol{\phi}, \sigma_{\tau}^{2}, \rho, \tau_{0}, \tau_{-1}\right) \propto p\left(\sigma_{c}^{2}\right) \times\left(\sigma_{c}^{2}\right)^{-\frac{T}{2}} \mathrm{e}^{-\frac{1}{2\left(1-\rho^{2}\right) \sigma_{c}^{2}}\left(k_{1}-\frac{2 \rho \sigma_{c}}{\sigma_{\tau}} k_{2}+\frac{\rho^{2} \sigma_{c}^{2}}{\sigma_{\tau}^{2}} k_{3}\right)},
$$

where $p\left(\sigma_{c}^{2}\right)$ is the uniform prior specified above. This full conditional density of $\sigma_{c}^{2}$ is not a standard density and we sample from it using a Griddy-Gibbs step. That is, we evaluate the full conditional density on a fine grid, and obtain a draw from the density using the inverse-transform method (see, e.g., Kroese, Taimre, and Botev, 2011, pp. 45-47). Steps 4 and 5 can be similarly implemented by noting that

$$
\begin{aligned}
& p\left(\sigma_{\tau}^{2} \mid \mathbf{y}, \boldsymbol{\tau}, \boldsymbol{\phi}, \sigma_{c}^{2}, \rho, \tau_{0}, \tau_{-1}\right) \propto p\left(\sigma_{\tau}^{2}\right) \times\left(\sigma_{\tau}^{2}\right)^{-\frac{T}{2}} \mathrm{e}^{-\frac{1}{2 \sigma_{\tau}^{2}} k_{3}-\frac{1}{2\left(1-\rho^{2}\right) \sigma_{c}^{2}}\left(k_{1}-\frac{2 \rho \sigma_{c}}{\sigma_{\tau}} k_{2}+\frac{\rho^{2} \sigma_{c}^{2}}{\sigma_{\tau}^{2}} k_{3}\right)} \\
& p\left(\rho \mid \mathbf{y}, \boldsymbol{\tau}, \boldsymbol{\phi}, \sigma_{c}^{2}, \sigma_{\tau}^{2}, \tau_{0}, \tau_{-1}\right) \propto p(\rho) \times\left(1-\rho^{2}\right)^{-\frac{T}{2}} \mathrm{e}^{-\frac{1}{2\left(1-\rho^{2}\right) \sigma_{c}^{2}}\left(k_{1}-\frac{2 \rho \sigma_{c}}{\sigma_{\tau}} k_{2}+\frac{\rho^{2} \sigma_{c}^{2}}{\sigma_{\tau}^{2}} k_{3}\right)},
\end{aligned}
$$


where $p\left(\sigma_{\tau}^{2}\right)$ and $p(\rho)$ are the priors for $\sigma_{\tau}^{2}$ and $\rho$ respectively.

Lastly, to jointly sample $\tau_{0}$ and $\tau_{-1}$, note that we can write $\boldsymbol{\alpha}=\mathbf{X}_{\boldsymbol{\delta}} \boldsymbol{\delta}$, where $\boldsymbol{\delta}=\left(\tau_{0}, \tau_{-1}\right)^{\prime}$ and

$$
\mathbf{X}_{\boldsymbol{\delta}}=\left(\begin{array}{cc}
2 & -1 \\
3 & -2 \\
\vdots & \vdots \\
T+1 & -T
\end{array}\right)
$$

It follows from (13) that the conditional distribution of $\boldsymbol{\tau}$ given $\mathbf{u}^{c}$ and other parameters is

$$
\left(\boldsymbol{\tau} \mid \mathbf{u}^{c}, \sigma_{c}^{2}, \sigma_{\tau}^{2}, \rho, \tau_{0}, \tau_{-1}\right) \sim \mathcal{N}\left(\mathbf{X}_{\boldsymbol{\delta}} \boldsymbol{\delta}+\frac{\rho \sigma_{\tau}}{\sigma_{y}} \mathbf{H}_{2}^{-1} \mathbf{u}^{c},\left(1-\rho^{2}\right) \sigma_{\tau}^{2}\left(\mathbf{H}_{2}^{\prime} \mathbf{H}_{2}\right)^{-1}\right) .
$$

Then, by standard regression results, we have

$$
\left(\tau_{0}, \tau_{-1} \mid \mathbf{y}, \boldsymbol{\tau}, \sigma_{c}^{2}, \sigma_{\tau}^{2}, \rho, \phi\right) \sim \mathcal{N}\left(\widehat{\boldsymbol{\delta}}, \mathbf{K}_{\boldsymbol{\delta}}^{-1}\right)
$$

where

$$
\begin{aligned}
\mathbf{K}_{\boldsymbol{\delta}} & =\mathbf{V}_{\boldsymbol{\delta}}^{-1}+\frac{1}{\left(1-\rho^{2}\right) \sigma_{\tau}^{2}} \mathbf{X}_{\boldsymbol{\delta}}^{\prime} \mathbf{H}_{2}^{\prime} \mathbf{H}_{2} \mathbf{X}_{\boldsymbol{\delta}} \\
\widehat{\boldsymbol{\delta}} & =\mathbf{K}_{\boldsymbol{\delta}}^{-1}\left(\mathbf{V}_{\boldsymbol{\delta}}^{-1} \boldsymbol{\delta}_{0}+\frac{1}{\left(1-\rho^{2}\right) \sigma_{\tau}^{2}} \mathbf{X}_{\boldsymbol{\delta}}^{\prime} \mathbf{H}_{2}^{\prime} \mathbf{H}_{2}\left(\boldsymbol{\tau}-\frac{\rho \sigma_{\tau}}{\sigma_{y}} \mathbf{H}_{2}^{-1} \mathbf{u}^{c}\right)\right),
\end{aligned}
$$

where $\mathbf{V}_{\boldsymbol{\delta}}=\operatorname{diag}\left(V_{\tau}, V_{\tau}\right)$ and $\boldsymbol{\delta}_{0}=\left(\tau_{00}, \tau_{00}\right)^{\prime}$. 


\section{References}

N. Baştürk, C. Çakmakli, S. P. Ceyhan, and H. K. Van Dijk. Posterior-predictive evidence on US inflation using extended new Keynesian Phillips curve models with non-filtered data. Journal of Applied Econometrics, 29(7):1164-1182, 2014.

M. Banbura, D. Giannone, and L. Reichlin. Large Bayesian vector auto regressions. Journal of Applied Econometrics, 25(1):71-92, 2010.

T. Berger, G. Everaert, and H. Vierke. Testing for time variation in an unobserved components model for the U.S. economy. Journal of Economic Dynamics and Control, 2016. Forthcoming.

S. Beveridge and C. R. Nelson. A new approach to decomposition of economic time series into permanent and transitory components with particular attention to measurement of the 'business cycle'. Journal of Monetary Economics, 7(2):151 - 174, 1981.

Z. I. Botev, J. F. Grotowski, and D. P. Kroese. Kernel density estimation via diffusion. The Annals of Statistics, 38(5):2916-2957, 2010.

F. Canova. Bridging DSGE models and the raw data. Journal of Monetary Economics, $67: 1-15,2014$.

A. Carriero, G. Kapetanios, and M. Marcellino. Forecasting exchange rates with a large Bayesian VAR. International Journal of Forecasting, 25(2):400-417, 2009.

J. C. C. Chan. Moving average stochastic volatility models with application to inflation forecast. Journal of Econometrics, 176(2):162-172, 2013.

J. C. C. Chan and E. Eisenstat. Marginal likelihood estimation with the Cross-Entropy method. Econometric Reviews, 34(3):256-285, 2015.

J. C. C. Chan and I. Jeliazkov. Efficient simulation and integrated likelihood estimation in state space models. International Journal of Mathematical Modelling and Numerical Optimisation, 1(1/2):101-120, 2009.

P. K. Clark. The cyclical component of US economic activity. The Quarterly Journal of Economics, 102(4):797-814, 1987.

J. Geweke and G. Amisano. Hierarchical Markov normal mixture models with applications to financial asset returns. Journal of Applied Econometrics, 26(1):1-29, 2011. 
R. J. Gordon. The demise of US economic growth: Restatement, rebuttal, and reflections. Technical report, National Bureau of Economic Research, 2014.

A. L. Grant and J. C. C. Chan. A Bayesian model comparison for trend-cycle decompositions of output. Journal of Money, Credit and Banking, 2016. Forthcoming.

A. C. Harvey. Trends and cycles in macroeconomic time series. Journal of Business and Economic Statistics, 3(3):216-227, 1985.

A. C. Harvey and A. Jaeger. Detrending, stylized facts and the business cycle. Journal of Applied Econometrics, 8(3):231-231, 1993.

A. C. Harvey, T. M. Trimbur, and H. K. Van Dijk. Trends and cycles in economic time series: A Bayesian approach. Journal of Econometrics, 140:618-649, 2007.

R. J. Hodrick and E. C. Prescott. Postwar US business cycles: An empirical investigation. Carnegie Mellon University discussion paper, no. 451., 1980.

R. J. Hodrick and E. C. Prescott. Postwar US business cycles: An empirical investigation. Journal of Money, Credit, and Banking, pages 1-16, 1997.

G. Kamber, J. Morley, and B. Wong. Intuitive and reliable estimates of the output gap from a Beveridge-Nelson filter. UNSW Business School Research Paper No. 2016-09, 2016.

G. Koop. Bayesian Econometrics. Wiley \& Sons, New York, 2003.

G. Koop. Forecasting with medium and large Bayesian VARs. Journal of Applied Econometrics, 28(2):177-203, 2013.

D. P. Kroese and J. C. C. Chan. Statistical Modeling and Computation. Springer, New York, 2014.

D. P. Kroese, T. Taimre, and Z. I. Botev. Handbook of Monte Carlo Methods. Wiley \& Sons, New York, 2011.

S. Luo and R. Startz. Is it one break or ongoing permanent shocks that explains US real GDP? Journal of Monetary Economics, 66:155-163, 2014.

W. J. McCausland, S. Miller, and D. Pelletier. Simulation smoothing for state-space models: A computational efficiency analysis. Computational Statistics and Data Analysis, 55(1):199-212, 2011. 
J. C. Morley and J. Piger. The asymmetric business cycle. Review of Economics and Statistics, 94(1):208-221, 2012.

J. C. Morley, C. R. Nelson, and E. Zivot. Why are the Beveridge-Nelson and unobservedcomponents decompositions of GDP so different? Review of Economics and Statistics, 85(2):235-243, 2003.

P. Perron and T. Wada. Let's take a break: Trends and cycles in US real GDP. Journal of Monetary Economics, 56(6):749-765, 2009.

W. Polasek. The Hodrick-Prescott (HP) filter as a Bayesian regression model. Working Paper Series 46-11, The Rimini Centre for Economic Analysis, 2011.

M. W. Watson. Univariate detrending methods with stochastic trends. Journal of Monetary Economics, 18(1):49-75, 1986. 